\title{
Multicriteria mapping of stakeholder preferences in regulating nanotechnology
}

\author{
Steffen Foss Hansen
}

Received: 21 September 2009/Accepted: 18 June 2010/Published online: 30 June 2010

(C) Springer Science+Business Media B.V. 2010

\begin{abstract}
In order to facilitate stakeholder discussions on how to regulate nanotechnology, the opensource program multicriteria mapping (MCM) was used to structure 26 interviews with stakeholders in the USA. MCM offers a systematic part quantitative, part qualitative approach to clarify why some regulatory options (bans, moratoriums, voluntary measures, etc.) were deemed to be acceptable/unacceptable by various stakeholders and which criteria stakeholders used to evaluate the different regulatory options. Adopting an incremental approach and implementing a new regulatory framework was evaluated as the best options whereas a complete ban and no additional regulation of nanotechnology were found to be the least favorable. Criteria applied differed substantially among stakeholders and included social, ethical, regulatory, environmental, and health issues. Opinions on future regulation seem far less polarized than expected and it seems that stakeholders would welcome a combination of voluntary measures, an incremental approach and forming of a new regulatory framework.
\end{abstract}

Electronic supplementary material The online version of this article (doi:10.1007/s11051-010-0006-3) contains supplementary material, which is available to authorized users.

S. F. Hansen $(\bowtie)$

Department of Environmental Engineering, NanoDTU,

Technical University of Denmark, Building 113,

2800 Kgs, Lyngby, Denmark

e-mail: sfh@env.dtu.dk
Keywords Nanotechnology - Regulatory options · Multicriteria mapping $\cdot$ Stakeholders ·

Societal dimensions

\section{Introduction}

A number of recent publications on governance of nanomaterials have pointed to stakeholder deliberation as a key element for nanotechnology to reach its full potential as well as secure democratic and transparent decision-making processes (IRGC 2006; Greenwood 2007). The open-source program termed multicriteria mapping (MCM) was used to structure 26 interviews with various stakeholders in the USA.

MCM offers a systematic part quantitative, part qualitative approach to clarify why various stakeholders deem some regulatory options acceptable/ unacceptable and which criteria stakeholders use to evaluate the different regulatory options (Stirling $2005 a, b) . M C M$ is one of many multiple criteria decision analysis (MCDA) methods. The common purpose of these methods is to evaluate and choose among different decision alternatives based on multiple criteria using systematic, structured and transparent analysis in contrast to "ad hoc" decisions (Linkov et al. 2006). A number of different MCDA methods exist following various optimization algorithms and varying in both the types of value information needed and in the extent to which they are dependent on computer software. Some 
techniques rank options whereas others identify a single optimal alternative and again others differentiate between acceptable and unacceptable alternatives (Linkov et al. 2007). See Linkov et al. (2007) for detailed introduction to various MCDA methods and their strengths and weaknesses.

Linkov et al. (2007) showed the theoretical applicability of MCDA to evaluate three hypothetical nanomaterials whereas Tervonen et al. (2009) recently used an outranking model termed Stochastic multicriteria acceptability analysis (SMAA-TRI) to group nanomaterials (e.g., C60, MWCNT, CdSe) in various risk classes (extreme, high, medium, low, and very low risk) for screening level risk assessments. Based on a literature review Tervonen et al. (2009) set forward a number of criteria, both in terms of nanoparticle properties as well bioavailability, bioaccumulation, and toxic potential. Quantitative criterion were either measured or based on expert judgments whereas qualitative criteria were established in terms of ordinal classes: 1 was the most favorable (least risk) value class, while 5 the least favorable (highest risk). Weight bonds were assigned to the various criteria by the authors, e.g., toxic potential $0.3-0.5$, bioavailability and bioaccumulation potentials $0.02-0.08$ and the rest of the criteria were assigned weight bounds of $0.05-0.15$. A cutting level within the range of 0.65-0.85 was then used to define the minimum sum of weights for the criteria that must be in concordance with the outranking relation to hold.

Expanding on the general framework of MCDA, Kuzma et al. (2008) used historical analysis, expert elicitation, and behavioral consensus to derive multidisciplinary criteria to guide and evaluating oversight of emerging technologies. Sixty-six initial criteria were identified from extensive literature reviews then expert elicitation has applied to narrow this down to 24 criteria such as impetus, stakeholder input, transparency, and health.

Key issues in relation to MCDA are: (1) who defines what the initial criteria are, (2) what the alternatives available to the decision-maker are, and (3) how the different criteria are translated into a numerical score in order to rank the different alternatives. In contrast to previous work on MCDA and nanomaterials, MCM overcomes these challenges by allowing each stakeholder to choose his/her own criteria as well as policy options eventually subject for the alternative evaluation. This reduces the potential biases introduced by having experts select criteria and having them assign weight of these.

MCM has previously been used to evaluate policy options in regard to genetically modified crops and obesity (Mayer and Stirling 2004; Millstone and Lobstein 2007), but this study presents the first MCM of stakeholder preferences in regulating nanotechnology.

I find that stakeholder opinions of how to move forward in regard to regulation of nanotechnology seem to be far less polarized than expected. In general there was agreement about the best policy options being an incremental approach followed by forming and implementing a new regulatory framework although stakeholders used a wide range of diverting criteria to evaluate the different options.

\section{Methods}

The interviews were completed in a 3-month period between May and August, 2007 prior to the U.S. Environmental Protection Agency's initiation of their voluntary Nanoscale Materials Stewardship Program (U.S. EPA 2007). A total of 53 stakeholders were identified and 26 agreed to be interviewed. For a distribution of these stakeholders into overall perspectives see Fig. 1.

Stakeholders were identified and contacted through a two-step process. First, contacts were taken with stakeholders and specialists (NGOs, academics, regulators, industry, etc.) that had publicly expressed their views on whether or not and how to regulate nanotechnology. Second, all interviewees were asked

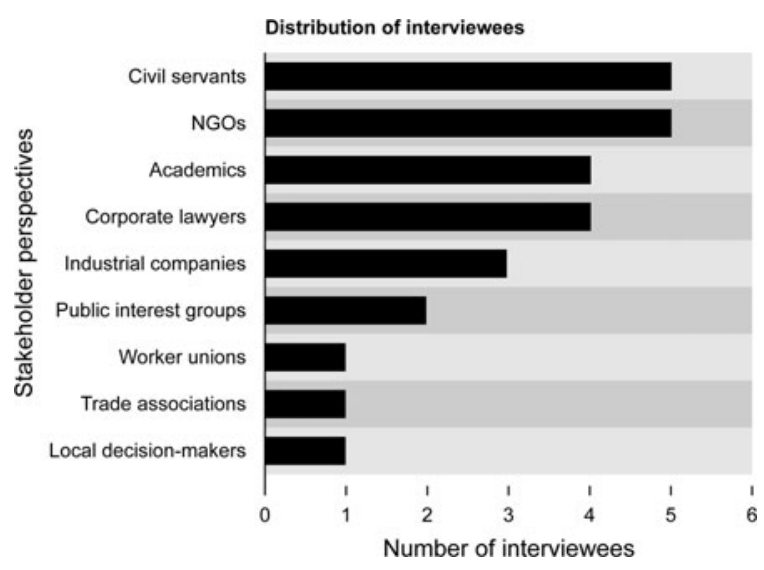

Fig. 1 Distribution of stakeholders into overall perspectives 
to help identify additional stakeholders relevant to the investigation. The name of the interviewee is kept anonymous in order to ensure that the interviewees could freely speak their views based on their personal experience and institutional background without fear of being identified or held accountable at a later point in time. Interviews were completed in a mutual understand of the fact that MCM provides a snapshot of what the perspectives are at the time of the interview and that they do not necessarily reflect current positions which might have changed in light of new evidence and insight.

A number of predefined policy options on how to regulate/not regulate nanotechnology had to be evaluated by all interviewees (see Table 1A). These options have been identified through a literature study and represent a wide range of views and hence the level of detail differs (European Commission 2004; NPPTAC 2005; Davies 2006; FOE 2006). The list is not meant to be a complete list, but rather a starting point for discussion.

The interviews and the analysis followed the guidelines provided by Stirling (2005a, b). The stakeholder interview was done face-to-face and followed a five-step sequence:

1. First the interviewees were asked to comment on the various policy options one at the time in regard to whether they felt a given option was acceptable/not acceptable, good or bad and whether there were policy options that they felt were missing from the list. If additional options were identified by the interviewee, these were added to the list of policy options.

2. Second, the interviewee was asked to identify and define the criteria (such as protection of public health and the environment, economical development, etc.) and principles (e.g., no use of animal tests) by which they had accessed the acceptability of the various options. "Criteria" were defined as factors that the interviewee had in mind when choosing between, or comparing, the pros and cons of different options whereas a principle may reflect a fundamental personal, institutional, ethical standpoint under which certain options are entirely ruled out.

3. Once the criteria had been identified, the interviewee was asked to evaluate the relative performance of the different policy options on a numerical scale $(0-100)$ under each of the criteria one-by-one. 0 representing the worst and 100 the best relative performance. In order to allow for uncertainty in the estimation the MCM software allows one to give a range rather than a single number. MCM also allows one to make worst-case and best-case assumptions (e.g., assuming no and full participation in a voluntary program) and give a low and high score. Throughout this scoring process the interviewee was asked to explain the value or range assigned to the option and assumptions about worst- and best-case scenarios were noted and interviews were taped in order to ensure accuracy.

4. Then the interviewee was asked to assign values concerning the relative importance of the different criteria for instance if an interviewee had four different criteria, they could weight those 10, 15, 25 , and $50 \%$, respectively.

5. The final rank was discussed in order to make sure that they did indeed reflect the view of the interviewee.

Given this information the MCM software generates a final ranking taking the different options, their performance scores and criteria weightings into account. The final ranking of each option is calculated as a weighted sum, i.e., for each participant it is derived by multiplying the participant's positive and pessimistic scoring of each option under a specific criterion (step 3) by the weight percentages assigned to that criterion (step 4), repeating this process for each of the participant's criteria and then summing these products.

\section{Results}

Each interviewee was asked to give their immediate perspective on the pros and cons of each of the predefined policy options and whether they found that any additional policy options should be added to the list of options. 22 additional policy options were identified. Most of these involved a combination or a slight rewording of the predefined options, e.g., substituting "hazard assessment" with "risk assessment" or adding the word "mandatory". Other stakeholders identified and defined fairly comprehensive regulatory options (see Table 1B and supplementary information). 
Table 1 Predefined policy options and additional options identified

A. Predefined policy options

1. Ban nanotechnology: ban R\&D and commercialization of nanotechnology and nanomaterials

2. Ban some nanotechnologies and materials: ban some branches of nanotechnologies and materials based on hazard assessment

3. Moratorium on R\&D and commercialization: decreeing a moratorium on nanotechnologies R\&D and/or commercialization for targeted engineered nanomaterials until safety has been tested

4. Moratorium of commercialization: moratorium of commercialization of all nanomaterials until safety has been tested

5. Relying on voluntary measures: relying on industry voluntary measures-The Government will set up a forum for exchange of information on the properties of nanomaterials and health and safety issues based on voluntary industry reporting and testing

6. Forming and implementing a new regulatory framework: launching a comprehensive, in-depth regulatory process specific to nanotechnologies that aims at developing an entirely new legislative framework that tries to take all the widely different nanomaterials and applications into consideration

7. The incremental approach: launching an incremental process using existing legislative structures-e.g., dangerous substances legislation, classification and labeling, cosmetic legislation, etc.- to the maximum, revisiting them, and, when appropriate only, amending them. This includes issuing recommendations, commissioning studies, promoting risk assessment throughout the life cycle of a nanotechnology; encouraging actions of existing institutions; supporting the setting up an observatory of nanotechnologies; initiating a minimalist, appropriate and proportionate regulatory intervention setting up a framework within which stakeholders can participate in shaping the course of nanotechnologies

8. No additional regulation needed: the current regulatory framework is considered adequate to protect humans and the environment from risks of nanotechnology and nanomaterials and there is no need for adaptation or additional regulation

B. Additional policy options identified

1. Forming a new regulatory framework plus moratorium on commercialization

2. Ban some specific nanomaterials based on risk assessment

3. Proactive precautionary incremental approach $^{\mathrm{a}}$

4. Environmental management systems on nanotechnology

5. Voluntary program plus incremental approach

6. Reactive incremental approach ${ }^{\mathrm{c}}$

7. Implement a voluntary program and rely on current legislation at present and development of a new regulatory framework down the road

8. Voluntary environmental program plus incremental approach plus new regulatory framework

9. Combination of a moratorium of commercialization plus forming and implementing a new regulatory framework

10. Combination of a moratorium of commercialization plus forming and implementing a new regulatory framework based on nonvertebrate testing ${ }^{\mathrm{d}}$

11. Incremental approach plus ban some branches of nanotechnologies and materials based on hazard assessment

12. Aggressive incremental approach $^{\mathrm{e}}$

13. Ban some nanotechnologies and materials or moratorium R\&D and commercialization combined with either forming and implement-ting a new regulatory framework or an incremental approach

14. Voluntary measures plus incremental approach

15. Preventive incremental approach $^{\mathrm{f}}$

16. Proportional incremental approach ${ }^{\mathrm{g}}$

17. Free marked based on insurance policies

18. Insurance and reinsurance based policies

19. Liability based policies

20. Incremental approach combined with publicly available industry-generated environmental, health and safety information ${ }^{\mathrm{h}}$

21. Evidentiary proportional approach combined with a non-step wise incremental approach ${ }^{\mathrm{i}}$

22. Voluntary measures and increased agency discretionary authorities while the current regulatory system is being revised ${ }^{\mathrm{j}}$

${ }^{a-j}$ See supplementary information for further definition of this option 
Criteria were defined as factors that the interviewee had in mind when choosing between, or comparing, the pros and cons of different options. Whereas most found it fairly easy to list the criteria by which they evaluate the different options, they generally struggled with clearly defining these criteria. One participant, for instance, defined transparency in decision-making process as "transparency in decision-making about the risk management in companies and in their communication of these risks and in regulatory judgments". Examples of definitions of criteria such as "quickness", "practicality", and "empowerment of people" include: (1) "the ability of the regulations to be implemented quickly", (2) "how realistic is it to implement them in the current political climate?", and (3) "empowering people to help themselves and be informed", respectively. Criteria such as protection of public health and the environment, minimize risks, and maximize benefits were often not further defined (see supplementary information for a full list of the criteria listed and evaluated by the participants).

Once the criteria had been defined, the interviewee was asked to evaluate the relative performance of the different policy options on a numerical scale (0-100) under each of the criteria one-by-one. Zero representing the worst relative performance and a 100 the best. In order to allow for uncertainty in the estimation MCM allows the interviewee to give a range (e.g., 20-30) and to make worst- and best-case assumptions. The lowest values assigned to an option would then reflect the option considered under worstcase assumptions whereas the highest would reflect the same option considered under best-case assumptions. Throughout this scoring process the interviewee was asked to explain the value or range assigned to options and assumptions made. One interview had to be terminated at this stage of the interview as the participant realized that he/she had yet to develop a formalized opinion on the most preferred options. Others expressed some dislike with having to put a numerical estimate on something which they normally only discuss in qualitative terms. Others again found it challenging to have to look at all the options through all their criteria scoring and explaining the scoring of up to 72 combinations of policy options and criteria. Normally they would not have to explain their position in such depth. Six participants formulated ultimate principles defined as issues for which trade-offs were unacceptable. These included "protection of public health", "that the public and companies participate actively in the decision-making process", "it should be in the public interests", "burden of proof to show safety on industry", "due process", and "fair risk and benefit distribution". Instead of assigning a numerical score to these principles, it was noted whether the interviewee felt that each option was either "acceptable" or "unacceptable". MCM is an iterative process, so interviewees were free to return to review earlier steps of the process at any stage of the interview.

After scoring the various options, interviewees were asked to assign values concerning the relative importance of the different criteria for instance if an interviewee had four different criteria, they could weight them $25 \%$ each. Taking the different options, their performance scores and criteria weightings into account a final ranking was generated and discussed with the interviewee (see Fig. 2). During the discussion of the final rank one interviewee realized one of the defined criteria was actually a matter of principle, whereas another interviewee realized that an additional criterion was used in the weighting process.
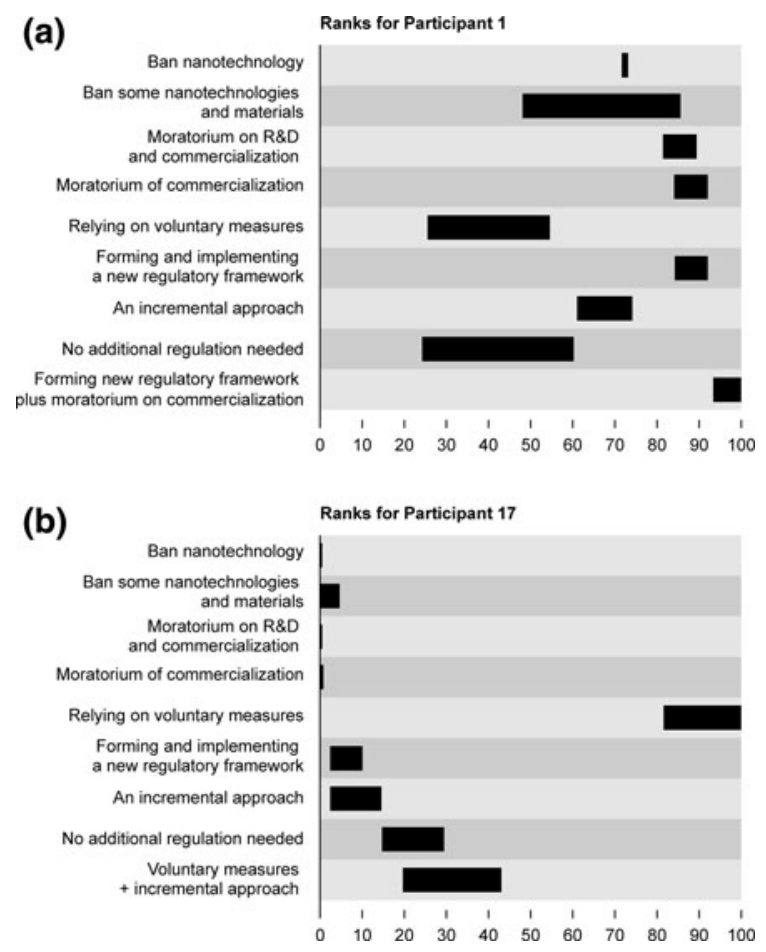

Fig. 2 Final ranking scheme generated for an academic (a) and the trade association representative (b) 
By the end all interviewees expressed satisfaction with their final ranking.

\section{Ranking of policy options}

Figure 2a shows the final ranking of an academic who use (1) unique benefits, (2) controllability of likely hazards, (3) life-cycle issues, and (4) environmental impact as criteria and who weighed them 27, 26, 26, and $21 \%$, respectively. The preferred option of this academic is a combination of the options of: forming and implementing a new regulatory framework while implementing moratorium of commercialization. Individually these two options were valued to be almost equally good, closely followed by having a moratorium of R\&D and commercialization.

Considering the options under a best- and worstcase scenario did not influence the ranking of the most preferred options, whereas it did for the policy options ranked worst. In a best-case scenario the worst options were evaluated to be relying on voluntary measures by this academic, followed by having no additional regulation and implementing an incremental approach. In a worst-case scenario worst options were having no additional regulation followed by relying on voluntary measures and implementing a ban of some nanotechnologies and materials. All of these options were evaluated to be worse than a complete ban of nanotechnology.

Figure $2 b$ shows an example of the final ranking of the trade association representative. This stakeholders criteria was "practicality", "societal and business benefits", and "reasonable in the view of current environmental, health and safety evidence" and these were weighed 8,45 , and $47 \%$, respectively. As the only participant, this stakeholder evaluated the option of relying on voluntary measures to prevail by far. The combination of relying on voluntary measures and having an incremental approach ranked second.

\section{Ranking of policy options by perspective}

Considering the ranking by the individual interviewee in regard to their overall perspective (i.e., Fig. 1) could provide valuable information about how various stakeholders evaluated the policy options as a
Fig. 3 Ranking of the various policy options across their overall perspectives $(\mathbf{a}-\mathbf{i})$ and the relative magnitudes of criteria weightings assigned to different issues under a selected perspective (I-IX). $\square$ Rank mean. $\mapsto$ Rank extreme

group. Figure $3 \mathrm{a}-\mathrm{i}$ shows the ranking of the various policy options across their overall perspectives.

In the best-case scenario the group of academics (see Fig. 3a) evaluated the forming and implementing of a new regulatory framework and an incremental approach to be the best options followed by having a moratorium of R\&D and commercialization. Least favorable options were found to be banning nanotechnology followed by relying on voluntary measures and no additional regulation. The evaluation of the performance of the various options considered under best and worst assumptions varies quite a bit within the academics interviewed as reflected in the length of the bars which is almost 30 points for all the options. Most variation evolves around the option of forming and implementing a new regulatory framework. This option was identified as the most favorable in a bestcase scenario and only fourth in a worst-case scenario, which is on a similar level as banning some nanotechnologies and materials and no additional regulation. The rank extreme bars show the level of the variability in the ranks assigned by different academics and indicates the lowest and the highest rank assigned to each option by any academic under any one criteria. There is an extensive overlap between the various options and hence any one option considered under best-case assumptions could potentially be ranked the highest, if compared with any of the alternative options considered using worst-case assumptions. For instance, in a best-case scenario banning nanotechnology could potentially be ranked first, if all other options are considered in a worst-case scenario.

Relying on voluntary measures was ranked 7 th and 8th in a best- and worst-case scenario, respectively, with values from 30 to 60 out of 100. Civil servants assigned similar values (e.g., 35-60) to this option, however, this resulted in it being ranked third (see Fig. 3b).

Civil servants, public interest groups and the worker union representative evaluated the forming and implementation of a new regulatory framework and an incremental approach as the best two options followed by relying on voluntary measures. Civil servants and public interest groups evaluated the worst options to be 
(a)

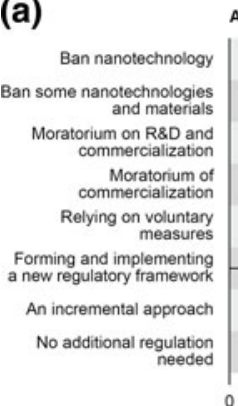

Academics ( $n=4)$

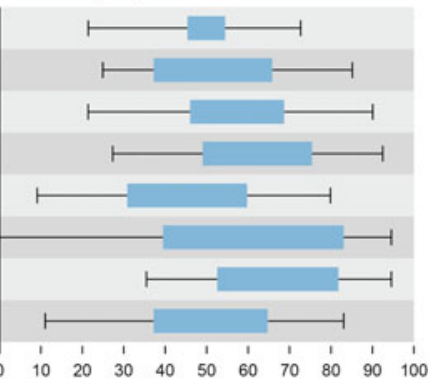

(b)

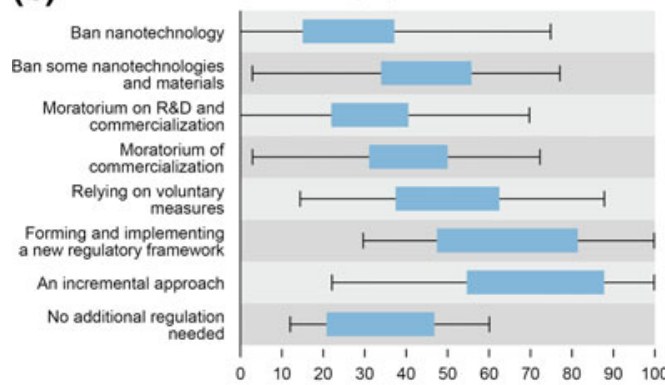

(c)

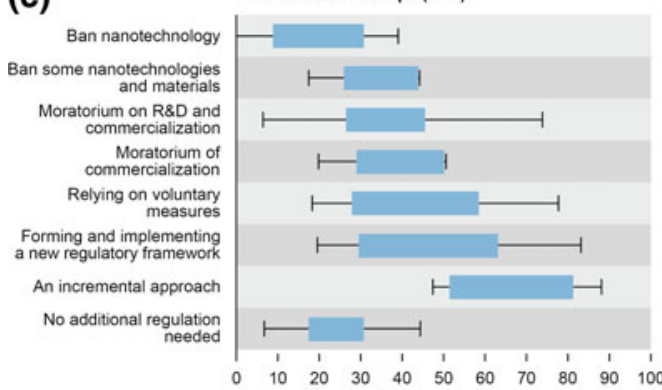

(d)

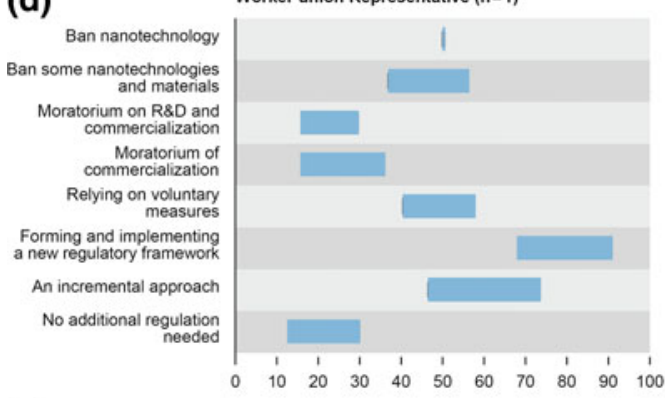

(e)

Industrial Company Representatives $(n=3)$

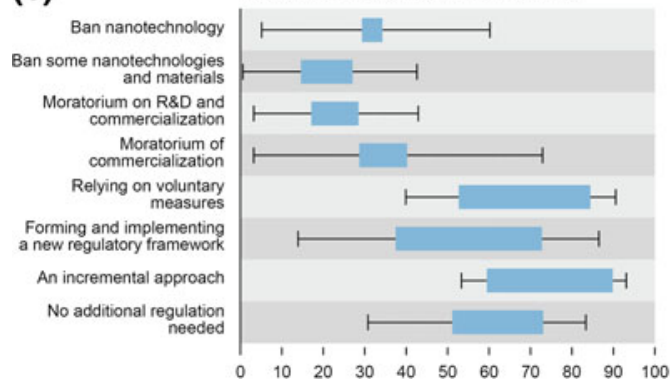

(I)

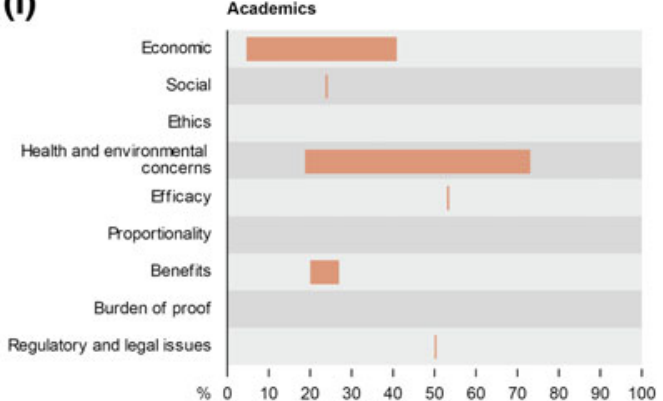

(II)

Civil Servants

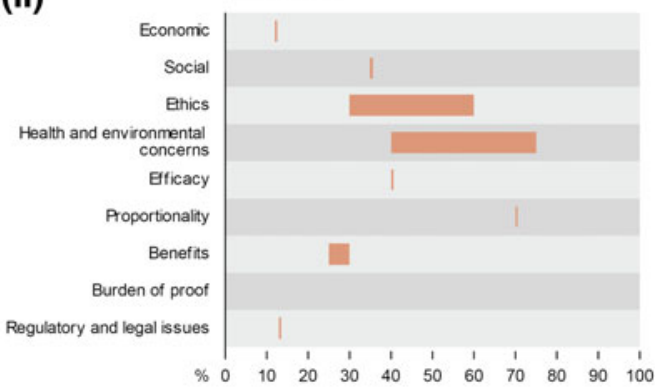

(III)

Public Interests Groups

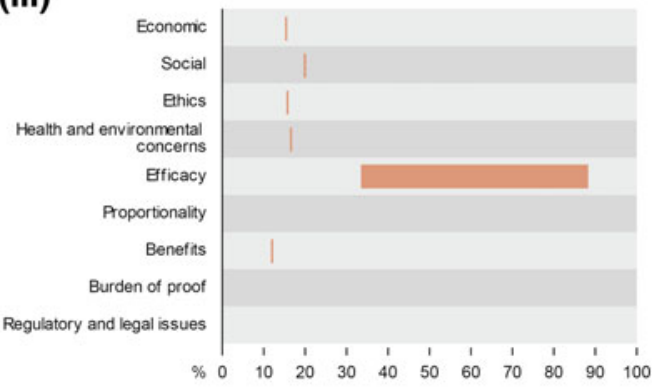

(IV) Worker Union Representative

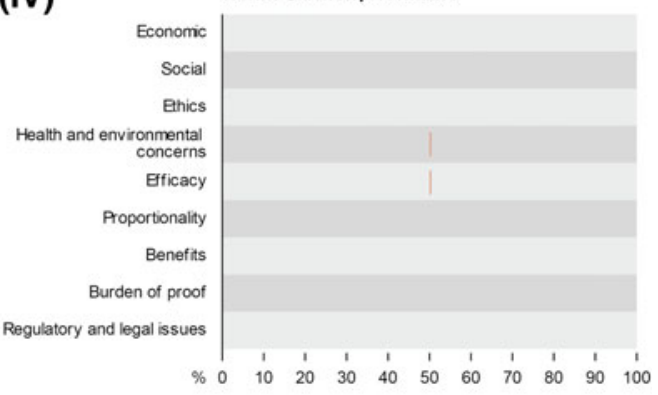

(V) Industrial Company Representatives

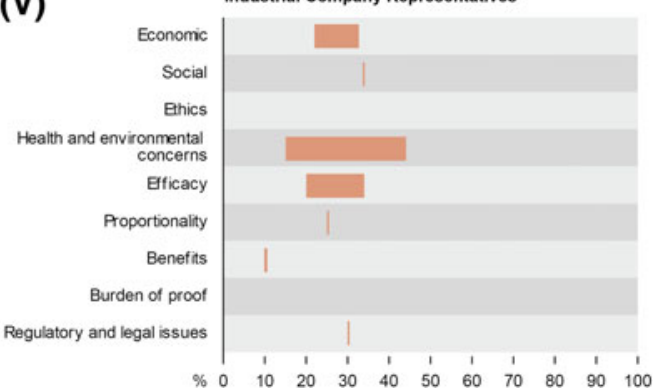


(f)

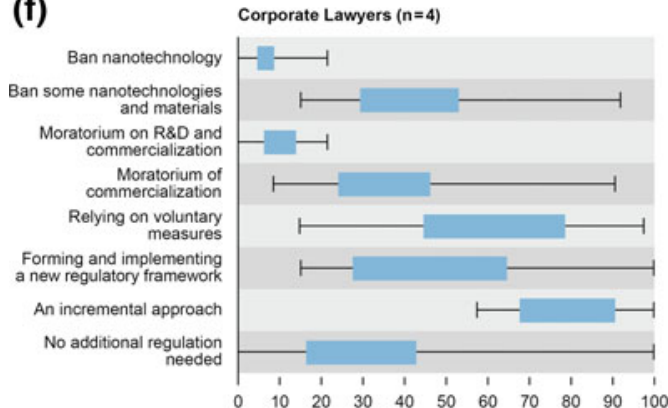

(g)

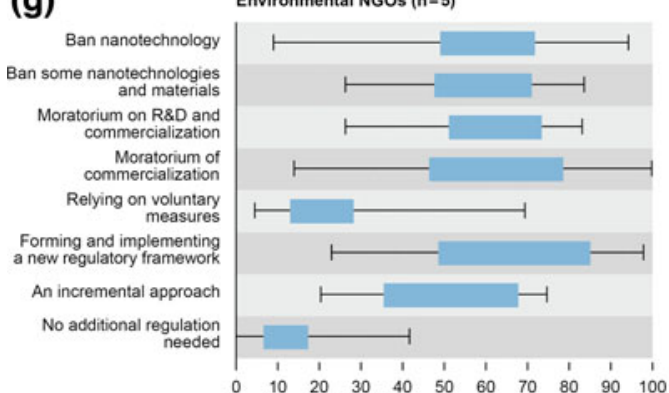

(h)

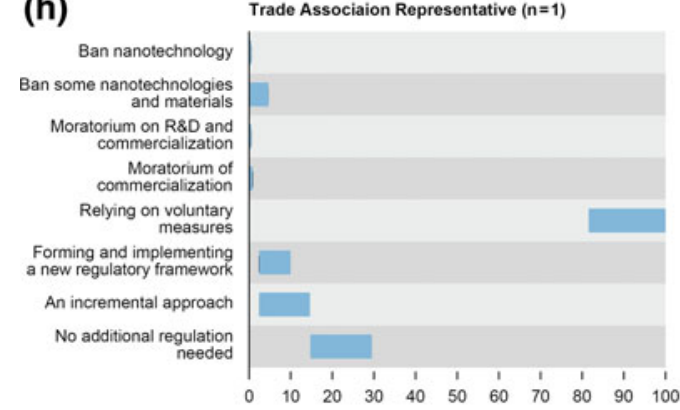

(i)

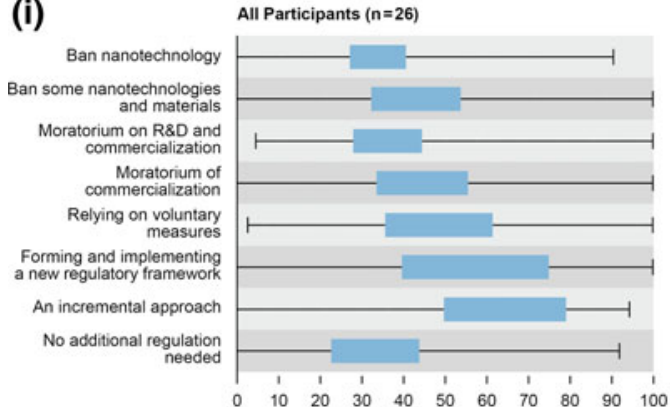

(VI)

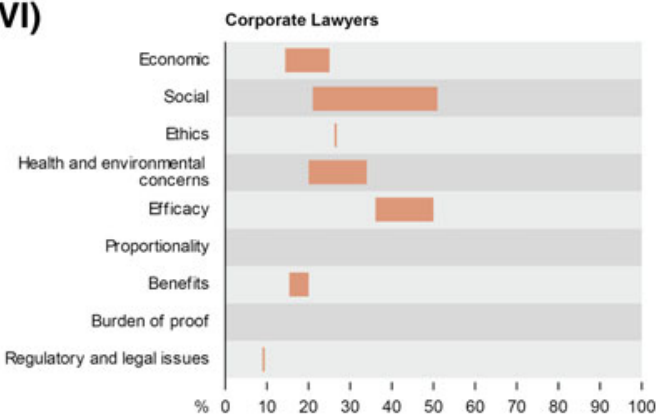

(VII)

Environmental NGOs

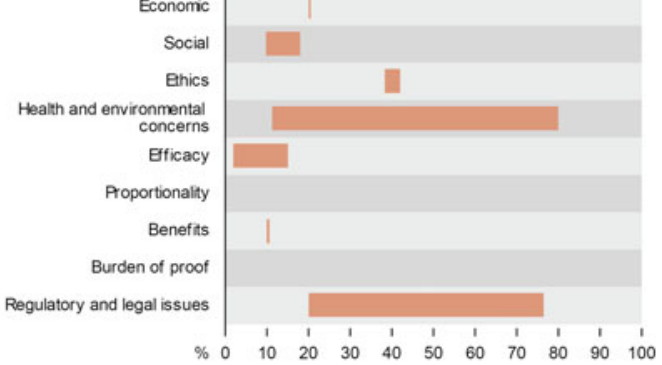

(VIII)

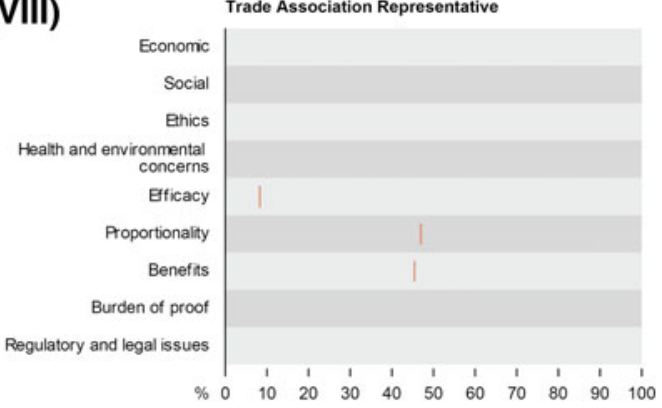

(IX)

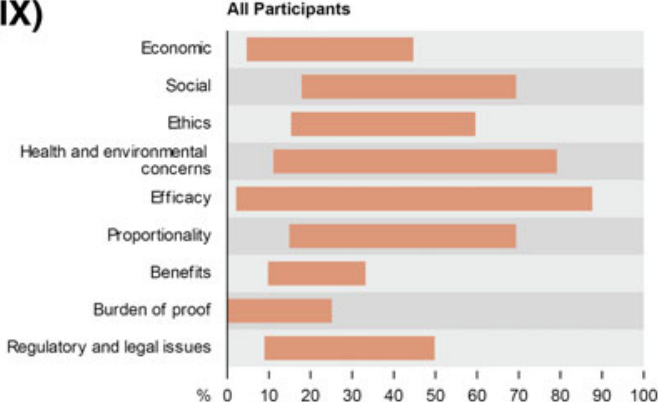

Fig. 3 continued

banning nanotechnology and no additional regulation, whereas the worker union representative found the worst options to be no additional regulation and the two versions of a moratorium.

The difference in performance of the various options when considered in a best- and worst-case scenario is quite high. The length of the bars for the

options ranked first and second by the civil servant is $\approx 40$ points. However, there is no overlap between options ranked highest and options ranked worst. For public interest groups the level of performance under best and worst assumptions varies similarly-especially for the top three options, however, again it has little impact on the overall ranking of the options. 
Industrial company representatives evaluated the incremental approach and relying on voluntary measures as the best options followed by no additional regulation needed and forming and implementing a new regulatory framework. The option of implementing a ban of nanotechnology scored higher than ban of some nanotechnologies and nanomaterials primarily due to the first being evaluated to protect human health and the environment more effectively. There is a high degree of overlap between the four top options and any one of them could potentially be ranked highest. The option of no additional regulation and forming and implementing a new regulatory framework was evaluated to be equally good under bestcase assumptions, whereas the former was evaluated to be far superior under worst-case assumptions.

The group of corporate lawyers also evaluated the best options to be implementing an incremental approach followed by relying on voluntary measures. A ban of nanotechnology and having a moratorium on $R \& D$ and commercialization ranked the lowest. The group of corporate lawyers disagreed largely on the applicability of the various options when considered under best- and worst-case scenario (25-55 points). However, it does not change the overall ranking of the various options much.

In contrast to the group of industrial company representatives and corporate lawyers, the option of implementing a moratorium on commercialization was ranked highest by the environmental NGOs under best-case assumptions, only second to forming and implementing a new regulatory framework. Worst options were evaluated to be no additional regulation and relying on voluntary measures. The length of the bars of the top six ranked options is quite large $(>25$ points) reflecting a large variation in regard to how well the options is evaluated to perform under worstand best-case assumptions. There is also a large degree of overlap between the top six options.

The only option considered viable by the trade association representative was relying on voluntary measures. This option was scored between 80 and 100 depending on whether the option was considered under worst- or best-case assumptions, whereas all other options were ranked below 30 .

Comparing the ranking of the various options by the stakeholder groups reveals that an incremental approach was ranked highest by civil servants, public interest groups, industrial company representatives and corporate lawyers both under best- and worstcase assumptions. Academics ranked this option first under worst-case assumptions and second under bestcase assumptions, respectively. The worker union representative had it ranked second under both scenarios. In contrast, environmental NGOs ranked this option only sixth. The option of forming and implementing a new regulatory framework was ranked highest by the worker union representative and second by public interest groups under both bestand worst-case assumptions whereas it was ranked first by academics and environmental NGOs under best-case assumptions only. Industrial company representatives and the trade association representative evaluated it to be only the third/fourth best option.

The option of relying on voluntary measures also generally ranked high and was ranked first by the trade association representative, second by the industrial company representatives and the corporate lawyers and third by the civil servants. Academics and NGOs, on the other hand, evaluated this option either worst or second to worst depending on whether the option was considered under best- and worst-case assumptions such as for instance full or limited participation by companies in a voluntary program.

The option to ban nanotechnology was ranked worst by the civil servants, public interest groups, corporate lawyers and the trade association representative whereas the group of academics evaluated it to be the worst option only in a best-case scenario. The option of no additional regulation was evaluated to be the least favorable option by the environmental NGOs and the worker union representative and second to worst by the public interest groups. The option of having a moratorium on $R \& D$ and commercialization was ranked second to worst by many stakeholder groups including corporate lawyers and representatives from the trade association, the worker union and the industrial companies.

The largest difference in ranking of the policy options can be observed between environmental NGOs and the representatives from the industrial companies and the trade association (see Fig. $3 \mathrm{~g}$, e, and $h$ ). The option of relying on voluntary measures and having no additional regulation are evaluated to be most favorable by the representative from the trade association, but the least favorable by the environmental NGOs whereas the three options ranked as most favorable by industrial company 
representatives were the options ranked the least favorable by the environmental NGOs.

When considering the ranking of all the options by all participants collectively the best options were evaluated to be an incremental approach followed by forming and implementing a new regulatory framework and relying on voluntary measures (see Fig. 3i). The ranking of these options vary greatly depending on whether they are considered under best- or worstcase scenario ( $>25$ points). Worst options were scored to be no additional regulation needed and ban of nanotechnology followed shortly by moratorium on $\mathrm{R} \& \mathrm{D}$ and commercialization and ban some nanotechnologies and materials and moratorium on commercialization. Under worst-case assumptions the option of no additional regulation needed is ranked lower than banning nanotechnology, which is due to the latter option being evaluated to address concerns about human health and environment more efficiently than having no additional regulation. Except for the option of implementing incremental approach there is a potential overlap between all the other options.

\section{Criteria and assigned weights}

97 different criteria were used by the interviewees (see supplementary information) with "Protection of human health and environment" being mentioned most often (6), followed by "Practicality" and "Transparency in the decision-making process" (3) (see Fig. 4).

The applied criteria could be divided into a number of clusters such as environmental health and safety concerns and economical, social and regulatory, and legal issues (see Fig. 4).

Criteria predominately fall into health and environmental concerns (27\%) followed by concerns about efficacy (14\%) and criteria that could be classified as benefits, economical, social, regulatory, and legal issues (10-13\%).

Figure 3I-IX displays the relative magnitudes of criteria weightings assigned to different issues under a selected perspective and should not be interpreted as an indication of the degree of difference in weightings attached by different participants to each issue. The length of the bars can be explained by both the differences in weightings and the number of participants defining criteria in the issues concerned. For instance, if the weighting bar displays no range at all, it means that only one participant developed this single criterion for the issue in question while the other participants excluding this issue altogether.

A similar ranking of best and worst policy options was found among academics, civil servants, public interest groups, corporate lawyers, and representatives from industrial companies and a worker union (see Fig. 3a-f), which could be at least partly explained by the criteria they find to be important and the weight participants gave to these (Fig. 3I-VI).

Health and environmental concerns was mentioned by more than one participant in five out of six stakeholders groups and the weight given to this one criterion ranged from around 15 up to $75 \%$ (see Fig. 3I-V, VII). Criterions related to efficacy were common between corporate lawyers, public interest groups and representatives from industrial companies and the worker union when they were asked to evaluate the various policy options. Several academics and industrial company representatives also put emphasize on economic issues.

The criterions used by NGOs and the trade association representative to evaluate the various policy options differed widely (see Fig. 3VII, VIII) which could help to explain their dissimilar ranking of the options (see Fig. $3 \mathrm{~g}, \mathrm{~h}$ ). Several NGOs put most weight on criteria that fell into the categories of health and environmental concerns and regulatory, legal, social and ethical issues, the trade association representative used criteria that fell into the categories of proportionality and benefits.

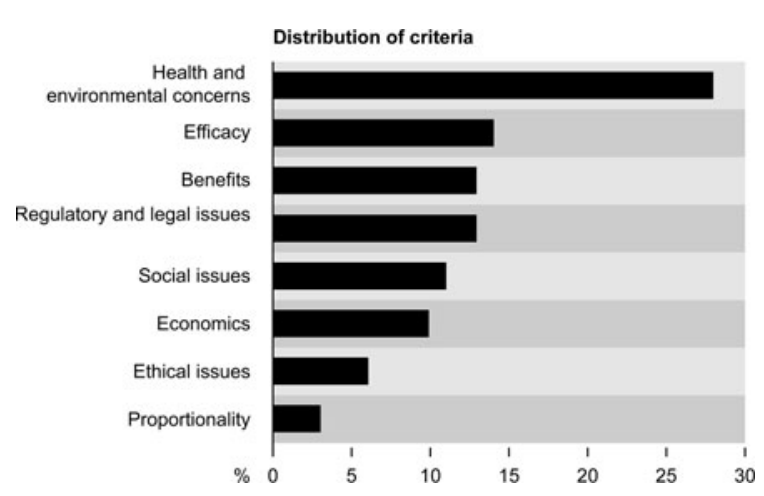

Fig. 4 Distribution of criteria classified according to clusters 


\section{Perspectives}

Although the results of the MCM provide some indication, firm conclusions about stakeholders preferences should be made with caution due to the small of interviewees overall (i.e., 26) and specifically in regard to small number of stakeholders representing various perspectives, e.g., academics, trade organizations, etc. Some of these perspectives are very broadly defined in this MCM analysis, e.g., academic. There is evidence that there is a great variation in the risk perception of nanomaterials of among various groups of academics (Powell 2007) and future work should go into investigating how these differences in risk perception transfer into a MCM analysis of preferable policy options.

Assuming for a moment that the 26 stakeholders subject for this MCM analysis qualify as a representative sample of the diverging views on acceptable/ non-acceptable policy options in regard to regulating nanomaterials, the results of the MCM analysis offers a number of interesting future perspectives. Most notably the fact that although the criteria on which stakeholders evaluate the various policy options differ substantially, a high level of agreement was observed among stakeholders about the most favorable predefined policy options being relying on voluntary measures, an incremental approach, and forming and implementation of a new regulatory framework. Of the predefined options, 24 out 26 ranked one of these three options the highest (data not shown). Several stakeholders actually suggested the possibility of implementing a combination of the three options. This indicates that a continuously overlapping combination of these three policy options could potentially outline a future political process that would be welcomed by most stakeholders. This would also be a potential compromise between the two most extreme stakeholder positions, i.e., environmental NGOs versus the trade association representative. The first step of such a potential future process (i.e., relying on voluntary measures) was evaluated highest by the trade association representative whereas combining this with an incremental approach was evaluated second overall. Environmental NGOs ranked relying on voluntary measures very low, but evaluated an incremental approach and forming and implementing a new regulatory framework highest. Environmental NGOs might be willing to accept relying on voluntary measures for a certain time-period while an incremental approach is being prepared.

The U.S. EPA has implemented voluntary measures with limited success (Maynard and Rejeski 2009). Therefore, it seems that the time is ripe to move onto a full implementation of an incremental approach and the initiation of discussions about the forming of a new regulatory framework. Attempts to address elements of an incremental approach have been made such as for instance recommendation and guidelines published by the U.S. National Institute for Occupational Safety and Health (NIOSH 2009), but these efforts constitutes a starting point only. A critical review and adaptations of the existing legislation is a fundamental element of an incremental approach that still has to be seriously addressed by policy-makers and agencies involved in the administration of, for instance, the Toxic Substances Control Act.

Acknowledgments The author would like to thank graphic designer Lisbeth Brusendorff, software developer Toby Champion and Professor Andy Stirling. Thanks are also due to the many stakeholders who agreed to be interviewed. He cannot thank them by name as he promised them anonymity.

\section{References}

Davies JC (2006) Managing the effects of nanotechnology. Project on emerging nanotechnologies. Woodrow Wilson International Center for Scholars, Washington, DC

U.S. EPA (2007) Nanoscale materials stewardship program and inventory status of nanoscale substances under the toxic substances control act; notice of availability. http:// www.epa.gov/fedrgstr/EPA-TOX/2007/July/Day-12/t13558. htm. Accessed 02 Jun 2009

European Commission, Health and Consumer Protection Directorate General (2004) Nanotechnologies: a preliminary risk analysis on the basis of a workshop. European Commission, Brussels

FOE (2006) Nanomaterials, sunscreens and cosmetics: small ingredients, big risks. Friends of the Earth Report (FoE), May 2006 (co-written by FoE Australia and US). http://nano.foe.org.au/filestore2/download/99/Nano\%20 cosmetics\%20report\%20-\%20web.pdf. Accessed 21 Sep 2009

Greenwood M (2007) Thinking big about things small: creating an effective oversight system for nanotechnology. Project on Emerging Nanotechnologies Woodrow Wilson International Center for Scholars, Washington, DC

IRGC (2006) White paper on nanotechnology risk governance. International Risk Governance Council, Geneva 
Kuzma J, Paradise J, Ramachandran G, Kim J-A, Kokotovich G, Wolf SM (2008) An integrated approach to oversight assessment for emerging technologies. Risk Anal 28(5): $1197-1219$

Linkov I, Satterstrom FK, Kiker G, Seager TP, Bridges T, Gardner KH, Rogers SH, Belluck DA, Meyer A (2006) Multicriteria decision analysis: a comprehensive decision approach for management of contaminated sediments. Risk Anal 26(1):61-78

Linkov I, Satterstrom FK, Steevens J, Ferguson E, Pleus RC (2007) Multi-criteria decision analysis and environmental risk assessment for nanomaterials. J Nanopart Res 9: 543-554

Mayer S, Stirling A (2004) GM crops, for good or bad? Those who choose the questions, determine the answers. EMBO Rep 5(11):1021-1024

Maynard A, Rejeski D (2009) Too small to overlook. Nat Nanotech 460:174

Millstone E, Lobstein T (2007) The PorGrow project: overall cross-national results, comparisons and implications. Obes Rev 8(Suppl. 2):29-36
National Pollution Prevention and Toxics Advisory Committee (2005) Overview document on nanoscale materials. U.S. Environmental Protection Agency, Washington, DC

NIOSH (2009) Approaches to safe nanotechnology managing the health and safety concerns associated with engineered nanomaterials. Department of Health and Human Services Centers for Disease Control and Prevention National Institute for Occupational Safety and Health

Powell M (2007) New risk or old risk? How scientists' standpoints shape their nanotechnology risk frames. Health Risk Soc 9(2):173-190

Stirling A (2005a) Multi-criteria mapping: a detailed interview manual. Version 4.4. May 2005. SPRU: University of Sussex

Stirling A (2005b) Multi-criteria mapping: a detailed analysis manual. Manual Version 2.2 2nd December 2005. SPRU: University of Sussex

Tervonen T, Figueira J, Steevens J, Kim J, Linkov I (2009) Risk-based classification system of nanomaterials. J Nanopart Res 11:757-766 